\title{
The relationship between gel mesh and particle size in determining nanoparticle diffusion in hydrogel nanocomposites
}

\author{
Paige J. Moncure, Zoe C. Simon, Jill E. Millstone, and Jennifer E. Laaser* \\ Department of Chemistry, University of Pittsburgh, Pittsburgh, PA \\ E-mail: j.laaser@pitt.edu
}

Phone: (412)383-0125

\begin{abstract}
The diffusion of poly(ethylene glycol) methyl ether thiol (PEGSH) functionalized gold nanoparticles (NPs) was measured in polyacrylamide gels of various crosslinking densities. The molecular weight of the PEGSH ligand and particle core size were both varied to yield particles with hydrodynamic diameters ranging from 7 to $21 \mathrm{~nm}$. Gel mesh size was varied from approximately 36 to $60 \mathrm{~nm}$ by controlling the crosslinking density of the gel. Because high molecular weight ligands are expected to yield more compressible particles, we expected the diffusion constants of the NPs to depend on their hard:soft ratios (where the hard component of the particle consists of the particle core and the soft component of the particle consists of the ligand shell). However, our measurements revealed that NP diffusion coefficients resulted primarily from changes in the overall hydrodynamic diameter and not the ratio of particle core size to ligand size. Across all particles and gels, we found that the diffusion coefficient was wellpredicted by the confinement ratio calculated from the diameter of the particle and an estimate of the gel mesh size obtained from the elastic blob model. These results
\end{abstract}


suggest that the elastic blob model provides a reasonable estimate of the mesh size that particles "see" as they diffuse through the gel. This work brings new insights into the factors that dictate how NPs move through polymer gels and will inform development of hydrogel nanocomposites for applications such as drug delivery in heterogeneous, viscoelastic biological materials.

\section{Introduction}

Understanding nanoparticle (NP) diffusion in hydrogel materials impacts many fields including medicine, ${ }^{1,2}$ polymer physics, ${ }^{3,4}$ and materials science. ${ }^{5,6}$ For example, hydrogels have emerged as a promising medium for NP-based drug delivery due to their biocompatibility, ${ }^{7,8}$ tunability, ${ }^{9,10}$ and response to external stimuli. ${ }^{11,12}$ Additionally, hydrogels have a network structure that strongly resembles biological media, such as mucus, ${ }^{13}$ extracellular matrix, ${ }^{14,15}$ and actin networks. ${ }^{16}$ Due to these similarities, NP-hydrogel composites are an attractive model system for understanding the complexities of nanoparticle transport through biological networks. ${ }^{17}$ Developing a detailed understanding of NP diffusion in hydrogel systems will contribute to advances in NP based drug delivery, particularly in predicting pharmacokinetics. ${ }^{18,19}$

NP diffusion in hydrogels is complex, particularly as the size of the particle approaches the gel mesh size. A number of theoretical approaches have been used to understand how the relationship between particle size and gel mesh size predicts diffusion. ${ }^{20-27}$ Early models of NP diffusion in networks treat the polymer chains as immobile fibers with pores that particles

can diffuse through. ${ }^{20,21}$ These models were later modified to include interactions between the particle and the polymer, as well as polymer chain reptation, ${ }^{22}$ which is important for describing more concentrated systems. Recently, to describe confined systems in which the probe particle is the same size or slightly larger than the nanostructure of the material, Cai et al. proposed the hopping diffusion model where thermal fluctuations of the network strands 
allow for diffusion. ${ }^{24}$ These theoretical models consider particle diffusion at many different polymer concentrations and level of confinement of the NP, and lay the ground work for interpreting experimental studies.

One key assumption that is made in most models of NP diffusion is that the nanoparticle is a solid sphere. This simplification may not correctly describe most real-world NPs which can be made with a vast array of different types of materials including metals, ${ }^{28,29}$ polymers, ${ }^{30,31}$ and DNA. ${ }^{32,33}$ A number of recent studies have begun to look at how diffusion is affected by changes in the composition of the particle, and specifically NP compressibility and elasticity. Latreille et al. studied the diffusion of both "soft" polymeric nanoparticles (composed of poly(N-isopropylacrylamide), with a hydrodynamic radius ranging from 25-130 nm) and "hard" particles (composed of either polystyrene or gold, with a hydrodynamic radius ranging from 22-110 nm), and found that the diffusion coefficient of the soft nanoparticles through a polymeric medium was nearly two orders of magnitude faster than the diffusion coefficient of hard nanoparticles of the same size. ${ }^{34}$ This study and others ${ }^{35-38}$ demonstrate that there are differences in the diffusion of NPs composed of hard materials or soft materials.

In addition to the tunability of the core composition, NP surface ligand shells can also be modified. Surface chemistry directly affects the chemical and physical properties of NPs, and highly specialized, complex ligand shells are essential for applications such as NP targeting and drug delivery. ${ }^{39-44}$ Not only do ligands impact NP surface chemistry, but they add an additional level of complexity to the diffusive behavior of the particles by changing the hydrodynamic diameter and introducing ligand-matrix effects. The effect of grafted polymer chains on NP surfaces has received substantial attention in the context of polymer melts and solutions, ${ }^{35,45-51}$ but these systems have important differences from networks and gels. In solution and melt polymer nanocomposites, polymer chains are not crosslinked and are more dynamic, leading to interactions with NPs that affect NP diffusion. ${ }^{52}$ Changes in NP diffusion as a function of ligand shell properties (e.g. molecular weight, environment-facing 
functional groups) are not well understood in the context of covalently crosslinked hydrogels.

While it is clear that nanoparticle diffusion is dictated by the characteristics of the NP, the properties of the gel itself also affect diffusion. The study of NP diffusion in gels is complicated by the heterogeneous distribution of crosslinks within typical hydrogels, ${ }^{53}$ making it difficult to ascertain the relationship between NP size and mesh size in determining NP diffusion behavior. Mesh size, here defined as the physical distance between crosslinks, is dependent on the swelling of the material, as well as its molecular structure. However, because hydrogels typically contain both areas of high crosslinking density and areas of low crosslinking density, it is difficult to define a single mesh size that is reflective of the material as a whole. ${ }^{54}$ Various models and experimental approaches have been developed to estimate mesh size, each making different assumptions about the material. These approaches include consideration of the synthetic conditions for the synthesis of the hydrogel, as well as analytical methods including rheology, light scattering experiments, and electron microscopy. ${ }^{55}$ Each of these models can result in a different predicted mesh size because they are sensitive to different features of the gels and rely on different approximations to connect the mesh size to the physical properties. Therefore, it is necessary to evaluate multiple models of mesh size to determine which is most accurate for the material in question.

Here, we investigate the diffusion of poly(ethylene glycol) methyl ether thiol (PEGSH) functionalized NPs through a poly(acrylamide) hydrogel, taking into account both the properties of the NPs and the properties of the gels that can affect diffusion. We varied the MW of the bound PEGSH ligand, yielding two series of particles with constant core diameters (of either $2 \mathrm{~nm}$ or $5 \mathrm{~nm}$ ) and increasing hydrodynamic diameters (ranging from $7 \mathrm{~nm}$ to $21 \mathrm{~nm}$ ). Our studies yielded two distinct but related conclusions: first, by measuring the diffusion of these NPs in gels of various crosslinking densities (yielding mesh sizes of $36 \mathrm{~nm}$ to $60 \mathrm{~nm}$ ), we show that the hydrodynamic diameter, not the size of the NP core, is the predominant NP property dictating diffusion. Second, we evaluated the mesh size of the 
hydrogels using multiple models, and showed that the elastic blob model, which estimates mesh size based on rheological measurements, was the best predictor of the extent to which the gels confined the particles and limited their diffusion. Taken together, our work lays the foundation for understanding how the relationship between a two-component nanoscale probe and the nanostructure of a complex network can dictate diffusion, which in turn is important for modeling and predicting pharmacokinetics of NP based drug delivery.

\section{Experimental}

\section{Materials}

Gold (III) chloride trihydrate $\left(\mathrm{HAuCl}_{4} \cdot 3 \mathrm{H}_{2} \mathrm{O} \geq 99.9 \%\right)$, sodium citrate tribasic dihydrate, poly(ethylene glycol) methyl ether thiol ( $\mathrm{MW}=1 \mathrm{kDa}, 2 \mathrm{kDa}, 5 \mathrm{kDa})$, acrylamide ( $\geq 99 \%)$, ammonium persulfate $(98 \%), \mathrm{N}, \mathrm{N}^{\prime}$-methylenebisacrylamide $(\geq 99.5 \%), \mathrm{N}, \mathrm{N}, \mathrm{N}^{\prime}, \mathrm{N}^{\prime}-$ tetramethylethylethylenediamine, and Aldrich ColorSpec disposable NMR tubes were obtained from Sigma-Aldrich (St. Louis, MO). Mono Zelux CMOS camera (CS165MU), 12 mm Machine Vision Lens (MVL12M23), and C-mount Adapter (SM1A10) were all purchased from Thorlabs (Newton, NJ). All reagents were used as received unless otherwise indicated. NANOpure (Thermo Scientific, $\geq 18.2 \mathrm{M} \Omega$ ) water was used to prepare all aqueous solutions. Prior to use, all glassware and Teflon-coated stir bars were washed with aqua regia (3:1 ratio of concentrated $\mathrm{HCl}$ to $\mathrm{HNO}_{3}$ ) and rinsed with copious amounts of water prior to drying. Caution: aqua regia is highly toxic and corrosive and should only be used with proper personal protective equipment and training. Aqua regia should be handled only inside a fume hood. 


\section{Nanoparticle Synthesis and Characterization}

\section{5 nm Gold Nanoparticle Synthesis}

Gold nanoparticles were synthesized following a modified Murphy synthesis. ${ }^{56}$ In a $20 \mathrm{~mL}$ glass vial, $14.5 \mathrm{~mL}$ of water was combined with $500 \mu \mathrm{L}$ of an aqueous $20 \mathrm{mM}$ gold (III) chloride trihydrate solution and $500 \mu \mathrm{L}$ of an aqueous trisodium citrate solution. An ice cold $0.1 \mathrm{M} \mathrm{NaBH}_{4}$ solution was prepared. To the gold and citrate solution, $0.6 \mathrm{~mL}$ of $\mathrm{NaBH}_{4}$ solution was added while stirring. Upon addition, the solution color changed from clear to orange. The mixture was removed from the stir plate and allowed to age for 1 hour prior to ligand exchange.

To $10 \mathrm{~mL}$ of the as-synthesized $5 \mathrm{~nm}$ gold nanoparticles, $250 \mu \mathrm{L}$ of $0.1 \mathrm{M}$ PEGSH solution (MW 1 kDa, MW 2 kDa, or MW 5 kDa) was added while stirring. Upon addition there was a slight color change to a pink/orange color. Particles were aged on the benchtop for $1 \mathrm{~h}$ prior to purification.

Particles were separated from excess PEGSH and citrate ligand, metal, and salts using molecular weight cutoff centrifugal filters. Approximately $4.5 \mathrm{~mL}$ of each NP solution were transferred to Amiconß Ultra - 4 Ultracel@ $30 \mathrm{kDa}$ molecular weight cutoff centrifugal filters (Merck Millipore Ltd.) and were spun using an Eppendorf 5804R centrifuge with a swing bucket rotor (A-44-4, Eppendorf, Inc.) with a force of $4000 \mathrm{rcf}$ for $10 \mathrm{~min}$. The resulting concentrated particles (typically 50-100 $\mu \mathrm{L}$ in water) were diluted in the tube to a volume of $4 \mathrm{~mL}$ with water. The loose pellet was resuspended by gentle mixing using a pipette prior to re-centrifugation. This washing procedure was repeated 3 times.

\section{2 nm Gold Nanoparticle Synthesis}

Gold nanoparticles were synthesized following a previously reported synthesis. ${ }^{57}$ In a $20 \mathrm{~mL}$ glass vial containing a stir bar while stirring at $1125 \mathrm{rpm}, 4.29 \mathrm{~mL}$ of water was combined with $188 \mu \mathrm{L}$ of $10 \mathrm{mM}$ PEGSH (MW $1 \mathrm{kDa}$, MW $2 \mathrm{kDa}$, and MW $5 \mathrm{kDa}$ ). While stirring, 
$450 \mu \mathrm{L}$ of $20 \mathrm{mM} \mathrm{NaBH}_{4}$ was added and solution color changed from light yellow to dark brown. The capped vials were aged on the benchtop for $1 \mathrm{~h}$ prior to purification.

Particles were separated from excess PEGSH and citrate ligand, metal, and salts using molecular weight cutoff centrifugal filters. Approximately $4.5 \mathrm{~mL}$ of each NP solution were transferred to Amiconß Ultra - 4 Ultracel@ $10 \mathrm{kDa}$ molecular weight cutoff centrifugal filters (Merck Millipore Ltd.) and were spun using an Eppendorf 5804R centrifuge with a swing bucket rotor (A-44-4, Eppendorf, Inc.) with a force of 4000 rcf for 10 min. The resulting concentrated particles (typically 50-100 $\mu \mathrm{L}$ in water) were diluted in the tube to a volume of $4 \mathrm{~mL}$ with water. The loose pellet was resuspended by gentle mixing using a pipette prior to re-centrifugation. This washing procedure was repeated 5 times.

\section{Nanoparticle Core Size and Hydrodynamic Diameter Characterization}

A dilute solution of nanoparticles was made by adding $10 \mu \mathrm{L}$ of particles (at as-synthesized concentration, post ligand exchange) to $100 \mu \mathrm{L}$ water. A $7 \mu \mathrm{L}$ aliquot was dropcast onto a carbon type A 200 mesh copper grid. The sample was allowed to air dry for at least 5 hours and then dried under vacuum overnight. TEM characterization of the particle core sizes was performed on a Hitachi 9500 ETEM with a Gatan Orius camera (Petersen Institute of Nanoscience and Engineering, Pittsburgh, PA). Nanoparticle hydrodynamic radii were determined via dynamic light scatting (DLS) on a Mavern Zetasizer operating at a wavelength of $633 \mathrm{~nm}$ and a scattering angle of $90^{\circ}$.

\section{Gel Synthesis and Characterization}

\section{Polyacrylamide Gel Synthesis}

Aqueous solutions of acrylamide and bisacrylamide were prepared at the concentrations listed in Table 2. To each aqueous solution, ammonium persulfate $(10 \mu \mathrm{L}$ at $10 \mathrm{w} / \mathrm{v} \%$ per $\mathrm{ml}$ of solution) and TEMED (1 $\mu \mathrm{L}$ per $\mathrm{mL}$ of solution) were added and mixed thoroughly into solution to initiate crosslinking. Samples were immediately transferred to disposable NMR 
tubes and capped. Samples gelled within one hour of initiator addition and were allowed to equilibrate for 12 hours before NPs were deposited on top. Gel samples will be referred to based on their mesh size determined using small-amplitude oscillatory shear rheology, as described below and summarized in Table 2.

\section{Gel Characterization}

Rheometry was performed on an Anton Paar MCR 302 rheometer using a 40 mm sandblasted parallel plate geometry. Frequency sweeps were conducted at $0.5 \%$ strain from $100 \mathrm{rad} / \mathrm{s}$ to $0.1 \mathrm{rad} / \mathrm{sec}$ and an amplitude sweep was conducted from $0.01 \%$ to $100 \%$ shear strain at $10 \mathrm{rad} / \mathrm{s}$, to assess the linear viscoelastic regime. Gels for rheology experiments were cast in a mold with a thickness of $1.5 \mathrm{~mm}$. The gap height in all rheology experiments was set to $1.2 \mathrm{~mm}$.

\section{Diffusion Characterization and Analysis}

\section{Diffusion Experiment Setup}

Within 24 hours of gel synthesis, NPs were deposited on top of the gels. Disposable NMR tubes were filled with approximately $7 \mathrm{~cm}$ of the gel and approximately $2 \mathrm{~cm}$ of concentrated NP solutions (note: if NP solution is not sufficiently concentrated, deswelling of the gel can occur). In a given batch, 12 NMR tubes with the same gel solution were prepared. Two replicates of each of the six NP core and ligand combinations were analyzed. Each batch of a single gel type was repeated 3 times for a total of 6 replicates of each NP core, ligand and gel combination.

Upon sample preparation, all 12 NMR tubes were placed in a homemade NMR tube holder. The samples and holder were placed in front of a light pad in an optics bay with no

other light exposure. A CMOS camera and a home built Labview code were used to take one image of the samples every hour for 70 hours resulting in 70 total timestamped images. 


\section{Diffusion Experiment Data Processing}

Diffusion images were processed using a home built Matlab code to which images and their time stamps were uploaded. The first image was displayed and the linescan location was selected. The starting position of the linescan was at least $1.5 \mathrm{~cm}$ above the interface (in the NP solution) and the end position was selected to be at least $1.5 \mathrm{~cm}$ below the interface (in the gel), as shown by the orange line in Figure 2a. Linescans at the same position for each image were extracted by interpolation and converted from intensity to absorbance, as shown in Figure 2b. All linescans were fit to two single sided error functions below, as defined by Herriarachi: ${ }^{58}$

$$
F(x, t)= \begin{cases}\operatorname{erfc}\left(\frac{x-x_{0}}{2 \sqrt{D_{1} t}}\right), & x<x_{0} \\ \operatorname{erfc}\left(\frac{x-x_{0}}{2 \sqrt{D_{2} t}}\right), & x>x_{0}\end{cases}
$$

where $\mathrm{F}$ is the intensity as a function of time, $D_{1}$ is the NP diffusion coefficient within the NP solution, $D_{2}$ is the diffusion coefficient within the gel, $\mathrm{t}$ is time, $x$ is the distance from the interface of the gel and NP solution, and $x_{0}$ is the initial interface position. The linescan from the first image was used to determine the initial position of the interface between the NPs and gel, and $x_{0}$ was held constant in all subsequent fits. All following linescans were fit to Equation 1 (Fig. 2c), to give the effective diffusion coefficient $\left(D_{2}\right)$ of the nanoparticles in the gel for each image (Fig. 2d). The diffusion coefficients from 20 hours to 70 hours were finally averaged to give the sample's average diffusion coefficient.

\section{Results}

\section{Nanoparticle Size Characterization}

We began by investigating the impact of varying the NP ligand shell thickness on NP diffusion through a polyacrylamide hydrogel. First, the core size of the NPs was measured via transmission electron microscopy (TEM) (see Supporting Information). As shown in 
Table 1: Nanoparticle core size and hydrodynamic diameter characterization data

\begin{tabular}{l|lll|lll}
\hline AuNP & $\begin{array}{l}\text { Hydrodynamic } \\
\text { Diameter } \\
(\mathrm{nm})^{a}\end{array}$ & $\begin{array}{l}\text { Stokes- } \\
\text { Einstein } \\
\text { Diffusion } \\
\text { Co- } \\
\text { efficient } \\
\left(\mu m^{2} / \text { sec }^{a}\right.\end{array}$ & $\begin{array}{l}\text { Core Di- } \\
\text { ameter } \\
(\mathrm{nm})^{b}\end{array}$ & $\begin{array}{l}\text { Ligand } \\
\text { Shell } \\
\text { Thickness } \\
(\mathrm{nm})^{c}\end{array}$ & $\begin{array}{l}\text { Ligand } \\
\text { Contour } \\
\text { Length } \\
(\mathrm{nm})\end{array}$ & Core:Ligand $^{d}$ \\
\hline $2 \mathrm{~nm} 1 \mathrm{kDa}$ & 7.6 & 63.9 & $1.8 \pm 0.4$ & 2.9 & 8.0 & 0.31 \\
$2 \mathrm{~nm} 2 \mathrm{kDa}$ & 11.3 & 43.0 & $1.8 \pm 0.3$ & 4.8 & 15.8 & 0.19 \\
$2 \mathrm{~nm} 5 \mathrm{kDa}$ & 16.8 & 28.9 & $1.7 \pm 0.3$ & 7.6 & 39.9 & 0.11 \\
\hline $5 \mathrm{~nm} 1 \mathrm{kDa}$ & 11.9 & 40.8 & $4.7 \pm 0.6$ & 3.6 & 8.0 & 0.65 \\
$5 \mathrm{~nm} 2 \mathrm{kDa}$ & 14.5 & 33.5 & $5.0 \pm 0.6$ & 4.8 & 15.8 & 0.53 \\
$5 \mathrm{~nm} 5 \mathrm{kDa}$ & 20.6 & 23.6 & $4.7 \pm 0.5$ & 8.0 & 39.9 & 0.30 \\
\hline
\end{tabular}

${ }^{a}$ Determined via DLS of samples; ${ }^{b}$ Determined via TEM of samples (TEM micrographs are shown in Figure S2); ${ }^{b}$ Calculated by subtracting core size from hydrodynamic diameter and dividing by $2 ;{ }^{d}$ Calculated by dividing core size by two times the ligand shell thickness.

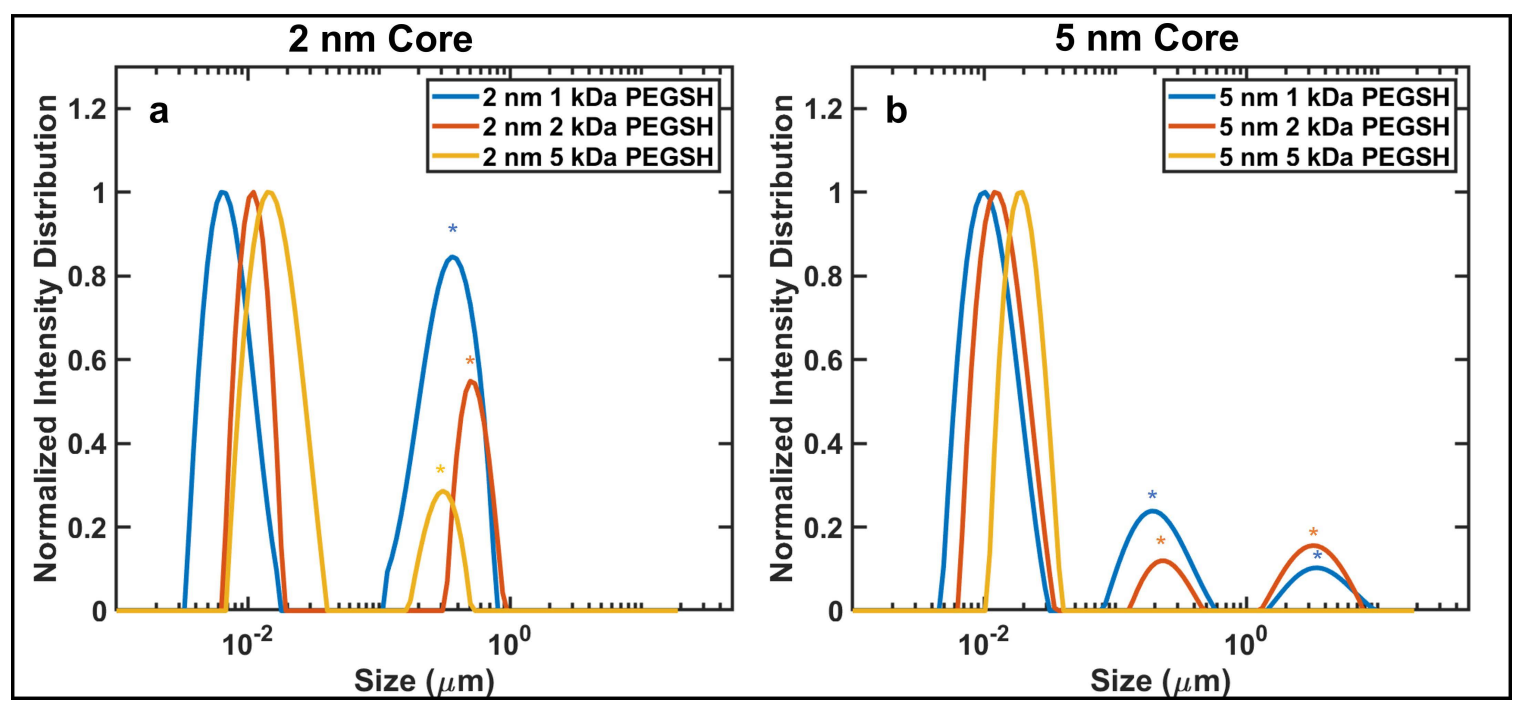

Figure 1: (a) Normalized DLS traces of the $2 \mathrm{~nm}$ core particle with $1 \mathrm{kDa}$ PEGSH, $2 \mathrm{kDa}$ PEGSH, and (b) $5 \mathrm{kDa}$ PEGSH and $5 \mathrm{~nm}$ core particle with $1 \mathrm{kDa}$ PEGSH, $2 \mathrm{kDa}$ PEGSH, and $5 \mathrm{kDa}$ PEGSH. Stars indicate peaks of detritus from molecular weight cutoff filters used to purify the samples. A DLS trace of filtered water demonstrating the detritus that comes off the filter is show in Figure S1. 
Table 1, the diameter of the cores of the particles was held constant at either approximately $2 \mathrm{~nm}$ or approximately $5 \mathrm{~nm}$. To vary the thickness of the ligand shell, each of the two particle core sizes were functionalized with a 1 kDa PEGSH, 2 kDa PEGSH, or 5 kDa PEGSH ligand. This resulted in a suite of 6 NP types: three with a core diameter of $2 \mathrm{~nm}$ (each one functionalized with either 1,2 , or $5 \mathrm{kDa}$ PEGSH), and three with a core diameter of $5 \mathrm{~nm}$ (each one also functionalized with either 1, 2, or $5 \mathrm{kDa}$ PEGSH). DLS was used to confirm the hydrodynamic diameter of each sample as shown in Figure 1. The hydrodynamic diameter of the particles systematically increased with both increasing core size and increasing MW of the ligand, resulting in particles with a hydrodynamic diameter from $7.6 \mathrm{~nm}$ (2 nm core with a $1 \mathrm{kDa}$ PEGSH) to $20.6 \mathrm{~nm}$ ( $5 \mathrm{~nm}$ core with $5 \mathrm{kDa}$ PEGSH). The thickness of the ligand shell in relation to the size of the core, here denoted core:ligand, varied between 0.11 (2 nm core with $5 \mathrm{kDa}$ PEGSH) and 0.65 (5 nm core with $1 \mathrm{kDa}$ PEGSH). The ligand shell thickness was compared to the ligand contour length, which is the theoretical length of the PEGSH chain if it were fully extended (calculated by adding the length of the PEG backbone, estimated as $0.35 \mathrm{~nm}^{59}$ ). The measured thickness of the $1 \mathrm{kDa}, 2 \mathrm{kDa}$, and $5 \mathrm{kDa}$ ligand shells was less than the contour length of the ligand, indicating that these ligands were partially collapsed. These data demonstrate that we were able to vary the NP ligand shell thickness while keeping the NP core size constant.

\section{Gel Size Characterization}

Table 2: Synthesized gel concentrations and estimates of mesh size and correlation length.

\begin{tabular}{ll|lll}
\hline $\begin{array}{l}\text { Acrylamide } \\
\begin{array}{l}\text { Concentration } \\
(\mathrm{w} / \mathrm{v} \%)\end{array}\end{array}$ & $\begin{array}{l}\text { Bis-acrylamide } \\
\text { Concentration } \\
(\mathrm{w} / \mathrm{v} \%)\end{array}$ & $\begin{array}{l}\text { Zero } \\
\text { Storage } \\
G_{(0)}^{\prime}{ }^{a}(\mathrm{~Pa})\end{array}$ & $\begin{array}{l}\text { Frequency } \\
\text { Modulus }\end{array}$ & $\begin{array}{l}\text { Gel Mesh Size via } \\
\text { Elastic Blob Theory } \\
(\mathrm{nm})\end{array}$ \\
\hline 3 & 0.0300 & 89.9 & 35.7 \\
3 & 0.0225 & 65.4 & 39.6 \\
3 & 0.0150 & 30.4 & 51.1 \\
3 & 19.2 & 59.7 \\
\hline
\end{tabular}


In addition to the properties of the NP, the crosslinking density of the gel was also varied - Four gels with varying amounts of the bisacrylamide crosslinker were synthesized. A $0.03 \mathrm{w} / \mathrm{v} \%$ bisacrylamide gel was selected as the gel with the highest crosslinking density because it was the limit at which all NPs had detectable diffusion coefficients in our setup. The $0.0113 \mathrm{w} / \mathrm{v} \%$ bisacrylamide gel was selected as the gel with the lowest crosslinking density because this was the lowest concentration at which samples reliably gelled. The zero-shear moduli of the gels were measured by small-amplitude oscillatory shear rheology, yielding values between 19.2 and $89.9 \mathrm{~Pa}$ (see Supporting Information). The mesh size of each gel was then estimated using elastic blob theory, which is appropriate for swollen networks. ${ }^{55}$ This model assumes that the polymers are well-described as elastically effective chains, and that the gel mesh size is equal to the size of the elastic blob $(\xi)$ given by

$$
\xi=\rho_{e l}^{-1 / 3}
$$

where $\rho_{e l}$ is equal to the number density of elastic blobs and is calculated from the zero frequency shear modulus $\left(G_{(0)}^{\prime}\right)$ via

$$
G_{(0)}^{\prime}=\rho_{e l} k_{B} T
$$

where $k_{B}$ is Boltzmann's constant and $\mathrm{T}$ is temperature. ${ }^{55}$ As shown in Table 2 the estimates of mesh size according to this model were on the nanometer scale for all gels, similar to the expected contour lengths of the strands between crosslinks (see Supporting Information). Additional models of mesh size, including the rubber elastic theory and the correlation length were evaluated as described in the Supporting Information.

\section{Nanoparticle Diffusion in Gels}

Following synthesis and characterization of the NPs and hydrogels, diffusion experiments were carried out, as described above. Briefly, NPs were deposited on top of hydrogels in 


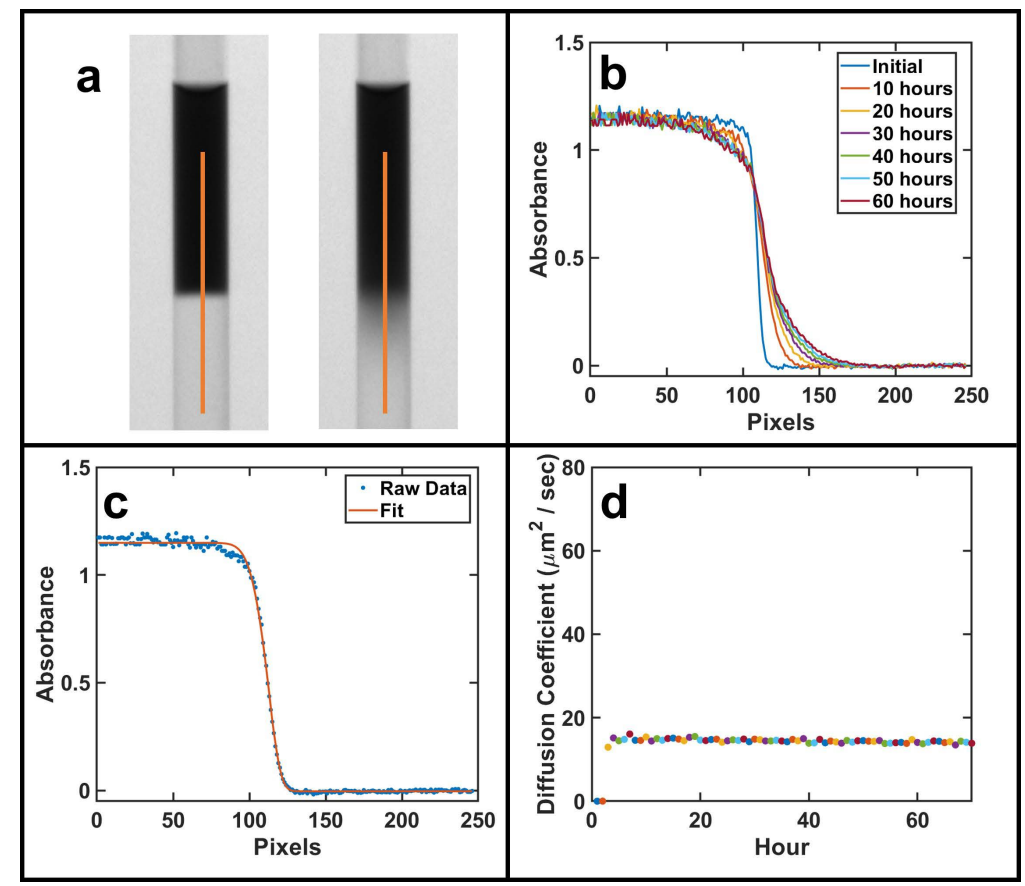

Figure 2: Images with NPs on top of hydrogels were taken every hour for 70 hours (a). Linescans were extracted from each image (b) and fit to Equation 1 (c). The diffusion coefficient $(\mathrm{d})$ was then calculated from the fit for each image.

NMR tubes and images were taken every hour over the course of the experiment as shown in Figure 2. Example images showing frame 1 (left) and frame 70 (right) from a sample containing $2 \mathrm{~nm} 1 \mathrm{kDa}$ NPs deposited on a gel with a mesh size of $35.7 \mathrm{~nm}$ are shown in Figure 2a. The orange line going from the NP solution into the gel shows the approximate location at which the linescans were extracted for each frame. Figure $2 \mathrm{~b}$ shows the absorbance as a function of position for every $10^{\text {th }}$ image. Each linescan went from high absorbance in the nanoparticle solution (pixels 0-100), to low absorbance in the gel (pixels 120-250). As time progressed the linescan between pixels 100 and 120 transitioned from a sharp drop-off to an increasingly gradual decay as the NPs diffused into the gel. Figure 2c illustrates a fit of a typical linescan to the error function shown in Equation 1. The resulting diffusion coefficients are plotted as a function of time in Figure 2d. As seen in this figure, the diffusion coefficient is essentially constant with little variation over time. 


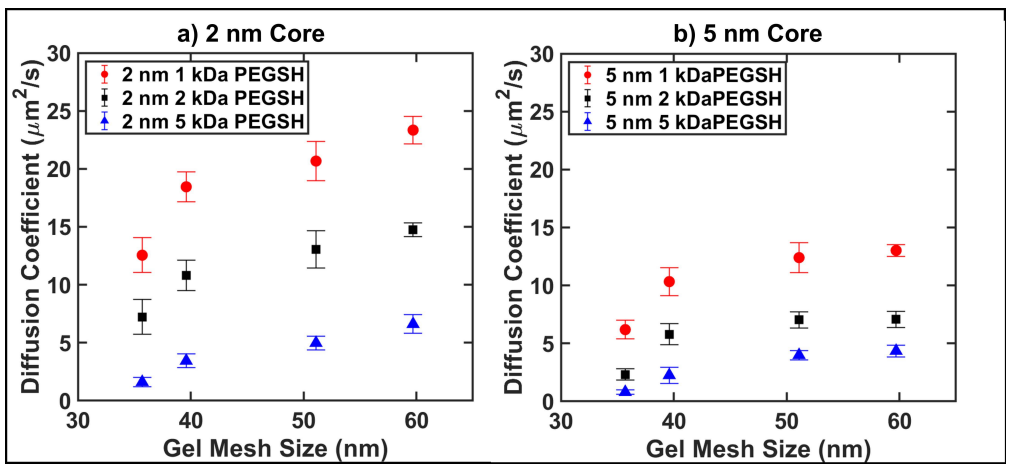

Figure 3: Diffusion coefficient as a function of gel mesh size for (a) $2 \mathrm{~nm}$ core particle and (b) $5 \mathrm{~nm}$ core particles.

Using this approach, the diffusion coefficients of each of the six NP types were measured in each of the four gels. A total of six replicates were averaged for each NP and gel combination. As shown in Figure 3, for all particles, the diffusion coefficient slowed as a function of increasing crosslinking density (corresponding to decreasing gel mesh size). Figure 3a shows the diffusion coefficients for all of the $2 \mathrm{~nm}$ core particles while Figure $3 \mathrm{~b}$ shows the diffusion coefficients for the $5 \mathrm{~nm}$ core particles, with the color and shape of the marker indicating the MW of the ligand shell. For all of the NP types, the diffusion coefficient increases with increasing gel mesh size. Additionally, for each gel mesh size, the diffusion coefficients of the particles decrease with increasing MW of the capping ligand. The decrease in diffusion coefficient with decreasing mesh size demonstrates that the NPs experience confinement in the gels and that the properties of the gel affect the diffusion of the NPs. Also, the decrease in diffusion coefficient with increasing thickness of the ligand shell is consistent with the increase in NP hydrodynamic diameter and demonstrates that the molecular weight of the ligand shell is affecting diffusion.

To understand the properties of the particle dictating diffusion, the diffusion coefficients were plotted as a function of the particle hydrodynamic diameter, as shown in Figure 4. For each of the samples, the diffusion coefficient decreases with increasing hydrodynamic diameter, regardless of the core size of the particle. The relationship between the diffusion coefficient and the hydrodynamic diameter follows the same trend as predicted by Stokes- 


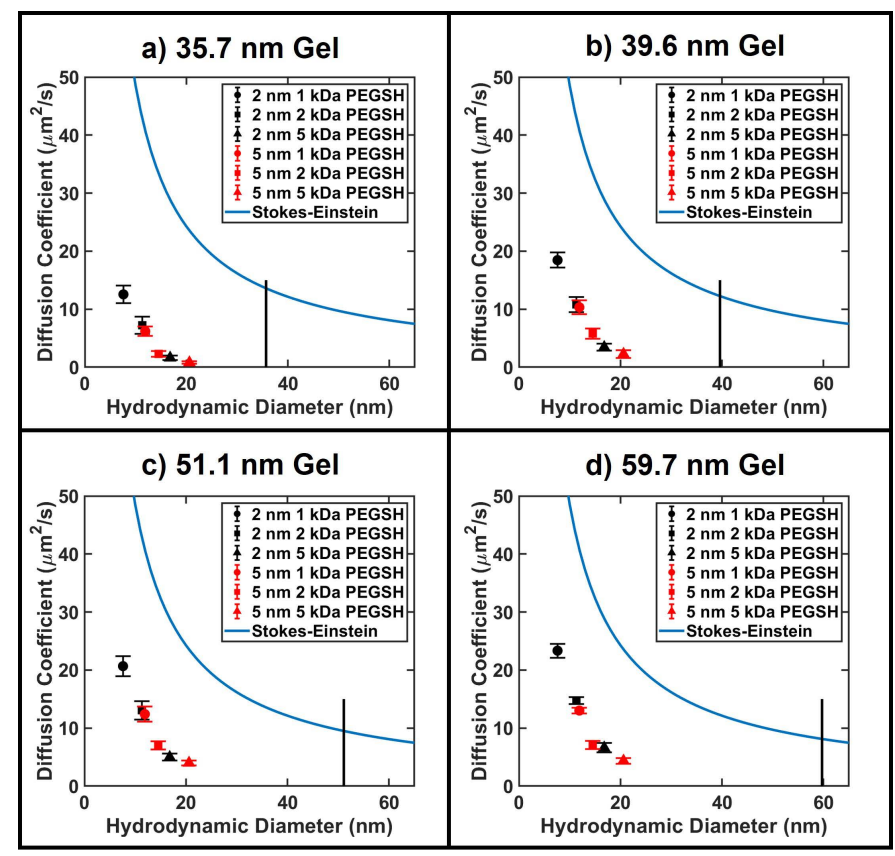

Figure 4: Diffusion coefficient in relation to hydrodynamic diameter of each gel type. Gels are denoted as their mesh size. The blue line represents the Stokes-Einstein relation for particles diffusing in water. The black vertical line indicates the gel mesh size.

Einstein, but is offset from the plotted Stokes-Einstein line, likely due to the increased effective viscosity of the gel relative to water. This figure indicates that diffusion is dependent on NP hydrodynamic diameter and not NP core size.

To further investigate the relationship between NP size and diffusion, the diffusion coefficient was plotted as a function of the core:ligand ratio for each gel mesh size, as shown in Figure 5. As reported in Table 1 the core:ligand ratio varies between 0.11 and 0.65 , with the $2 \mathrm{~nm}$ core NPs generally having a smaller core:ligand ratio than the $5 \mathrm{~nm}$ core NPs, with the exception of the $2 \mathrm{~nm} 1 \mathrm{kDa} \mathrm{NP}$ and the $5 \mathrm{~nm} 5 \mathrm{kDa}$ NPs which have roughly the same core:ligand ratio. Looking just at the $2 \mathrm{~nm}$ core particles, as the $\mathrm{MW}$ of the PEGSH ligand shell (and the hydrodynamic diameter) increases the core:ligand ratio and the diffusion coefficient of the particles decreases. This trend is also present for the $5 \mathrm{~nm}$ core particles but with larger core:ligand ratios. Comparison of particles with similar sizes but different core:ligand ratios, however, indicates that the core:ligand ratio is not a good 


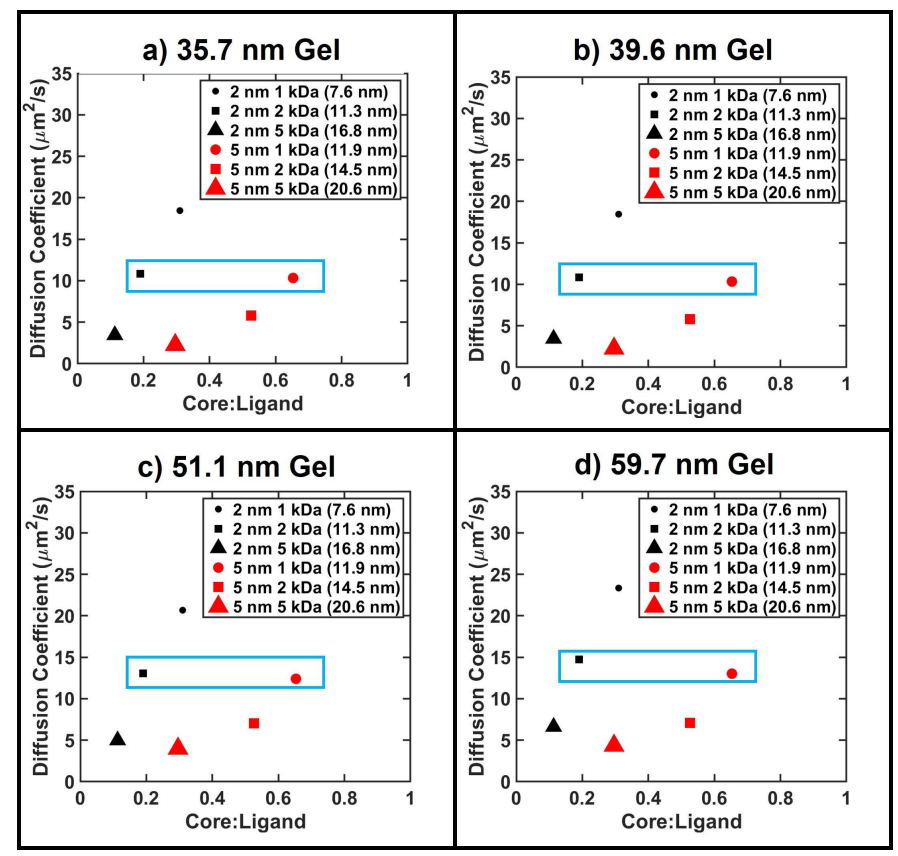

Figure 5: Diffusion coefficient as a function of core:ligand ratio for each gel type. Hydrodynamic diameter is shown by the relative size of the markers. Gels are denoted as their mesh size.

predictor of diffusion in these systems. This is illustrated clearly by the $2 \mathrm{~nm} 2 \mathrm{kDa}$ NPs (black square) and the $5 \mathrm{~nm} 1 \mathrm{kDa}$ NPs (red circle) highlighted by the blue box in Figure 5. These particles have different core:ligand ratios but similar hydrodynamic diameters, at $11.3 \mathrm{~nm}$ and $11.9 \mathrm{~nm}$, respectively. The diffusion coefficient for these particles in all gel types is roughly the same, indicating that the hydrodynamic diameter is dictating diffusion, not the core:ligand ratio.

After establishing that hydrodynamic diameter is the most relevant parameter for predicting diffusion in our system, we investigated how the relationship between particle size and gel mesh size affects diffusion by calculating the confinement ratio (particle diameter/gel mesh size) for each sample. The diffusion coefficients are plotted as a function of the confinement ratio calculated using the gel mesh size from the elastic blob theory in Figure 6. As seen in this figure, the diffusion coefficients decrease with increasing confinement ratio. The data from all four gels collapses onto a single curve when gel mesh is estimated via the elastic 


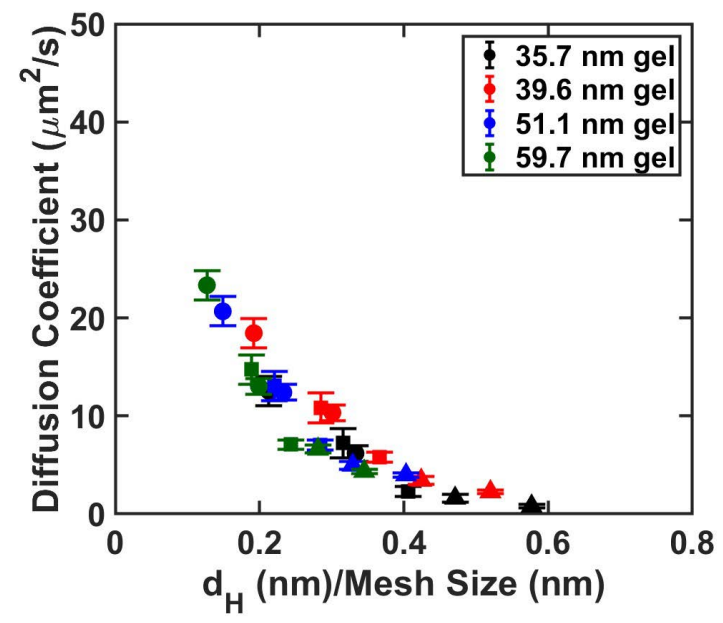

Figure 6: Diffusion coefficient as a function of confinement ratio (Hydrodynamic Diameter/Mesh Size), using mesh size calculated from elastic blob theory.

blob theory. By contrast, when the confinement ratio is calculated using gel mesh size based on contour length, correlation length, or mesh size calculated using rubber elastic theory (see Supporting Information), the data from the different gels do not collapse onto a single curve, indicating these models do not accurately reflect the features of the gel nanostructure relevant to NP diffusion.

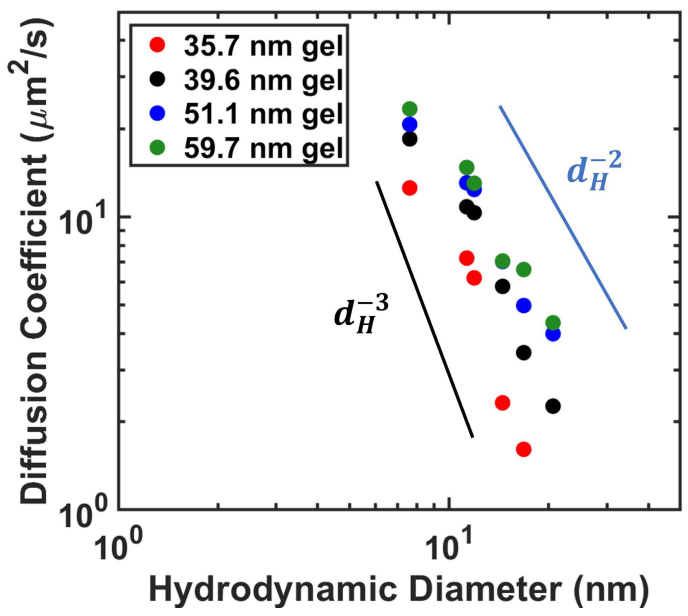

Figure 7: The diffusion coefficient for particles in each gel scales with hydrodynamic diameter $\left(d_{H}\right)$ with scaling laws between $d_{H}^{-2}$ (blue line) and $d_{H}^{-3}$ (black line), as described in the Supporting Information. 
Finally, to connect this experimental data to the theoretical work that has been done on these systems, we investigated the scaling between diffusion coefficient and hydrodynamic diameter. As the hydrodynamic diameter for all particles is smaller than the mesh size, we expect the diffusion to be inversely proportional to the minus third power of particle diameter. ${ }^{24}$ As shown in Figure 7, the diffusion coefficients in the gels with the smallest mesh size $(35.7 \mathrm{~nm})$ do roughly scale as $d_{H}{ }^{-3}$, while the diffusion coefficients in the gels with larger mesh sizes $(59.7 \mathrm{~nm}, 51.1 \mathrm{~nm}$, and $39.6 \mathrm{~nm})$ exhibit somewhat weaker scaling, with $D \sim d_{H}{ }^{-2}$. Exact scaling coefficients can be found in Table S2 (Supporting Information).

\section{Discussion}

In this work, we investigated the properties of both NPs and gels that dictate diffusion of NPs in hydrogel nanocomposites. We first hypothesized that the NP core size in relation to the gel mesh size would be the most appropriate predictor of diffusion. This hypothesis was based on the notion that the polymeric ligand shell is expected to be relatively compressible in relation to the inorganic NP core. ${ }^{45,47}$ Furthermore, previous publications have demonstrated that soft materials diffuse faster under confinement than hard materials. ${ }^{34,35}$ In this system, however, the nanoparticle diffusion was found to be dependent primarily on the overall hydrodynamic diameter of the particles, not the size of the core or the ratio between the core diameter and ligand shell thickness.

Two features of our experimental data demonstrate this point. If our initial hypothesis held, one would expect to see a separation between the diffusion coefficients of the small core NPs (Fig. 4, black symbols) and the large core NPs (Fig. 4, red symbols). However, in all gels, the diffusion coefficients for particles with the same hydrodynamic diameters were similar regardless of their core size, following the trend predicted by Stokes-Einstein (Figure 4). The key role of hydrodynamic diameter, rather than core:ligand ratio, is further supported when we compare the core:ligand ratio, the diffusion coefficient, and the hydro- 
dynamic diameter. Following our initial hypothesis, particles with lower core:ligand ratios should diffuse faster because they are more compressible. As seen in Figure 5, however, this was not the case. The $2 \mathrm{~nm} 2 \mathrm{kDa}$ NPs and the $5 \mathrm{~nm} 1 \mathrm{kDa}$ NPs have roughly the same hydrodynamic diameters with different core sizes and thus different core:ligand ratios, but have similar diffusion coefficients. This result indicates that diffusion is determined primarily by the hydrodynamic diameter, not by the core:ligand ratio. The insensitivity of the diffusion coefficient to the core:ligand ratio may indicate that the ligand shell in this system is effectively very rigid. However, testing this hypothesis will require directly varying the compressibility of the ligand shell, which is beyond the scope of the present work.

Another possible reason for the insensitivity to core:ligand ratio is that the ligand shell compressibility may not be relevant in this size regime. To test this hypothesis, we evaluated the scaling between the hydrodynamic diameter of the NPs and the diffusion coefficient for all gel types. As shown in Figure 7, the diffusion coefficient scales as approximately $D \sim d_{H}{ }^{-3}$ for the most tightly-crosslinked gel, as expected when the NP size is smaller than the gel mesh size, ${ }^{24}$ and exhibits only slightly weaker scaling for the more looselycrosslinked gels. This observation is in agreement with the relative sizes of our particles $(8 \mathrm{~nm}-21 \mathrm{~nm})$ in relationship to the gel mesh size $(36 \mathrm{~nm}-60 \mathrm{~nm})$. In this particular size regime, compressibility of the ligand shell may not significantly influence diffusion since the particles are not confined by the network cage. However, in larger size regimes such as those in which NPs undergo hopping diffusion between entanglement cages or network cages, ligand shell rigidity may be more relevant as the NP is more confined by the network.

Diffusion in hydrogel nanocomposites is not only dependent on the size of the NP but also the gel mesh size. Though mesh size is defined as the distance between crosslinks, determining this number in a randomly crosslinked gel is not straightforward in practice because of the random gelation process, which results in regions of very high crosslinking density and other regions of very low crosslinking density within the same sample. We found 
that the relationship between the particle size and the mesh size of the gels estimated from elastic blob theory accurately predicted the confinement (and diffusion) of the nanoparticles across all particles and gel sizes. The elastic blob model approximates the mesh size based on the size of the elastically effective chains, which is determined by the measured zero frequency shear modulus of the material. This model is useful for gels that are swollen as it assumes that the chains are extended and not freely rotating. The dependence of the diffusion coefficient on the confinement ratio, with the mesh size calculated from the elastic blob model, is shown in Figure 6. As seen in this figure, the data roughly collapses onto a single curve, indicating that the elastic blob model provides a reasonable description of what the diffusing NP feels.

While we found that the confinement of the NPs by the gel was well described by the mesh size from the elastic blob model, we also assessed three other models for mesh size that have been used in other studies. ${ }^{55,60}$ First, we evaluated the correlation length of the material, which is the theoretical distance between chains based on the volume fraction of polymer in the sample. As shown in Figure S6, when this model was used to calculate confinement ratio, the data did not collapse onto a single curve indicating that this estimate is not reflective of what is "felt" by a NP diffusing through the gel. Additionally, correlation length did not vary much between samples of different crosslinking densities, because the monomer concentration was kept constant across all gel syntheses. The next model evaluated was the mesh size estimated from the contour length of the chains between crosslinks. This model does not provide a realistic estimate of the mesh size because the assumption is made that the gel is fully homogeneous with equal crosslinking density throughout the material, which is not an accurate depiction of the randomly crosslinked gels in this study. The final model evaluated was the mesh size based on the rubber elastic theory. The mesh sizes predicted by this model were on the micrometer scale which is inconsistent with the measured diffusion coefficients of the NPs. This estimate is so large because this model treats the polymer 
chains between crosslinks as freely rotating, random Gaussian chains (modified by the Flory characteristic ratio) which is not appropriate for gels that are highly swollen, because the chains are highly extended and constrained by the covalent crosslinks. ${ }^{60}$ While these other models may be applicable to other gels, the relationship between the gel mesh size from the elastic blob model and the hydrodynamic diameter of the NP appeared to be the best predictor of diffusion in our system.

This work on understanding the roles of both NP size and gel mesh size in determining the diffusion of multi-component NPs in hydrogels lays the groundwork for developing models to understand NP diffusion for applications involving hydrogels. Many NP-based biological therapeutics rely on complex functionalization of the NP surface. Here, we have begun to understand how this ligand shell contributes to diffusion. Additionally, we have examined multiple models for estimating the nanostructure of the gel. We have found that in the system used in this work, the elastic blob model, which is based off of measured rheological properties of the material, is most accurate. Though this model was developed for synthetic materials, it has the potential to be useful for characterizing other non-synthetic viscoelastic materials, such as human mucus. Future experiments extending this study to size regimes where the nanoparticle is under more confinement from the gel will help further elucidate the physical factors that dictate the behavior of these systems.

\section{Conclusions}

The diffusion of PEG-functionalized NPs in polyacrylamide hydrogels was studied as a function of NP ligand length, NP core size, NP hydrodynamic diameter, and gel mesh size. As the gel mesh size was decreased, diffusion of the NPs also decreased, demonstrating that the NPs were diffusing under confinement. To understand what figure of merit was most relevant for predicting diffusion, NPs with different core sizes, ligand MWs, and hydrodynamic diameters were studied. Our results indicate that, in this system, NP diffusion is dependent 
only on the hydrodynamic diameter of the particle, not the size of the core or the ligand shell. We also examined multiple models for estimating gel mesh size, and found that the elastic blob theory provided the most accurate estimate of the local gel environment "felt" by the diffusing NP. Ultimately, by understanding what parameters of the gel and of the NP dictate diffusion, we aim to inform new models for improved predictions for NP transport and drug delivery in biologically-relevant viscoelastic environments. 


\section{Acknowledgement}

This work was supported by the University of Pittsburgh. We thank Professor Leanne Gilbertson at the University of Pittsburgh for generosity in using her DLS. This work was performed, in part, at the Nanoscale Fabrication and Characterization Facility, a laboratory of the Gertrude E. and John M. Petersen Institute of NanoScience and Engineering, housed at the University of Pittsburgh.

\section{Supporting Information Available}

Characterization data for nanoparticles and gels; supplemental analysis of mesh sizes and scaling relationships

\section{References}

(1) Merino, S.; Martín, C.; Kostarelos, K.; Prato, M.; Vázquez, E. Nanocomposite Hydrogels: 3D Polymer-Nanoparticle Synergies for On-Demand Drug Delivery. ACS Nano 2015, 9, 4686-4697.

(2) Satarkar, N. S.; Hilt, J. Z. Magnetic hydrogel nanocomposites for remote controlled pulsatile drug release. Journal of Controlled Release 2008, 130, 246-251.

(3) Wu, N.; Schultz, K. M. Microrheological characterization of covalent adaptable hydrogels for applications in oral delivery. Soft Matter 2019, 15, 5921-5932.

(4) Schultz, K. M.; Anseth, K. S. Monitoring degradation of matrix metalloproteinasescleavable PEG hydrogels via multiple particle tracking microrheology. Soft Matter 2013, 9, 1570-1579.

(5) Sershen, S. R.; Mensing, G. A.; Ng, M.; Halas, N. J.; Beebe, D. J.; West, J. L. Inde- 
pendent optical control of microfluidic valves formed from optomechanically responsive nanocomposite hydrogels. Advanced Materials 2005, 17, 1366-1368.

(6) Das, D.; Kar, T.; Das, P. K. Gel-nanocomposites:materials with promising applications. Soft Matter 2012, 8, 2348-2365.

(7) Wang, C.; Yokota, T.; Someya, T. Natural biopolymer-based biocompatible conductors for stretchable bioelectronics. Chemical Reviews 2021, 121, 2109-2146.

(8) Zhu, Y.; Haghniaz, R.; Hartel, M. C.; Mou, L.; Tian, X.; Garrido, P. R.; Wu, Z.; Hao, T.; Guan, S.; Ahadian, S.; Kim, H.-J.; Jucaud, V.; Dokmeci, M. R.; Khademhosseini, A. Recent Advances in Bioinspired Hydrogels: Materials, Devices, and Biosignal Computing. ACS Biomaterials Science \& Engineering 2021,

(9) Guo, Y.; Bae, J.; Fang, Z.; Li, P.; Zhao, F.; Yu, G. Hydrogels and Hydrogel-Derived Materials for Energy and Water Sustainability. Chemical Reviews 2020, 120, 76427707.

(10) Sun, X.; Agate, S.; Salem, K. S.; Lucia, L.; Pal, L. Hydrogel-Based Sensor Networks: Compositions, Properties, and Applications - A Review. ACS Applied Bio Materials 2021, 4, 140-162.

(11) Hoare, T. R.; Kohane, D. S. Hydrogels in drug delivery: Progress and challenges. Polymer 2008, 49, 1993-2007.

(12) Qiu, Y.; Park, K. Environment-sensitive hydrogels for drug delivery. Advanced Drug Delivery Reviews 2001, 53, 321-339.

(13) Joyner, K.; Song, D.; Hawkins, R. F.; Silcott, R. D.; Duncan, G. A.; Duncan, G. Designing viscoelastic mucin-based hydrogels. bioRxiv 2019, 1-11.

(14) Tibbitt, M. W.; Anseth, K. S. Hydrogels as extracellular matrix mimics for 3D cell culture. Biotechnology and Bioengineering 2009, 103, 655-663. 
(15) Theocharis, A. D.; Skandalis, S. S.; Gialeli, C.; Karamanos, N. K. Extracellular matrix structure. Advanced Drug Delivery Reviews 2016, 97, 4-27.

(16) Kouwer, P. H.; Koepf, M.; Le Sage, V. A.; Jaspers, M.; Van Buul, A. M.; EksteenAkeroyd, Z. H.; Woltinge, T.; Schwartz, E.; Kitto, H. J.; Hoogenboom, R.; Picken, S. J.; Nolte, R. J.; Mendes, E.; Rowan, A. E. Responsive biomimetic networks from polyisocyanopeptide hydrogels. Nature 2013, 493, 651-655.

(17) Saxton, M. J. Wanted: Scalable Tracers for Diffusion Measurements. The Journal of Physical Chemistry B 2014, 118, 12805-12817, PMID: 25319586.

(18) Dogra, P.; Butner, J. D.; Ruiz Ramírez, J.; li Chuang, Y.; Noureddine, A.; Jeffrey Brinker, C.; Cristini, V.; Wang, Z. A mathematical model to predict nanomedicine pharmacokinetics and tumor delivery. Computational and Structural Biotechnology Journal 2020, 18, 518-531.

(19) Kalwarczyk, T.; Kwapiszewska, K.; Szczepanski, K.; Sozanski, K.; Szymanski, J.; Michalska, B.; Patalas-Krawczyk, P.; Duszynski, J.; Holyst, R. Apparent Anomalous Diffusion in the Cytoplasm of Human Cells: The Effect of Probes' Polydispersity. The Journal of Physical Chemistry B 2017, 121, 9831-9837, PMID: 28956920.

(20) Ogston, A. G.; Preston, B. N.; Wells, J. D. on the Transport of Compact Particles Through Solutions of Chain-Polymers. Proc. Roy. Soc., London, Series a 1973, 333, $297-316$.

(21) Johansson, L.; Elvingson, C.; Löfroth, J. E. Diffusion and Interaction in Gels and Solutions. 3. Theoretical Results on the Obstruction Effect. Macromolecules 1991, 24 , 6024-6029.

(22) De Gennes, P. G. Dynamics of Entangled Polymer Solutions. II. Inclusion of Hydrodynamic Interactions. Macromolecules 1976, 9, 594-598. 
(23) Cai, L.-H.; Panyukov, S.; Rubinstein, M. Mobility of Nonsticky Nanoparticles in Polymer Liquids. Macromolecules 2011, 44, 7853-7863.

(24) Cai, L. H.; Panyukov, S.; Rubinstein, M. Hopping diffusion of nanoparticles in polymer matrices. Macromolecules 2015, 48, 847-862.

(25) Chen, Y.; Ma, R.; Qian, X.; Zhang, R.; Huang, X.; Xu, H.; Zhou, M.; Liu, J. Nanoparticle Mobility within Permanently Cross-Linked Polymer Networks. Macromolecules 2020, 53, 4172-4184.

(26) Sorichetti, V.; Hugouvieux, V.; Kob, W. Dynamics of nanoparticles in polydisperse polymer networks: From free diffusion to hopping. 2021,

(27) Dell, Z. E.; Schweizer, K. S. Theory of localization and activated hopping of nanoparticles in cross-linked networks and entangled polymer melts. Macromolecules 2014, 47, $405-414$.

(28) Sau, T. K.; Rogach, A. L.; Jäckel, F.; Klar, T. A.; Feldmann, J. Properties and applications of colloidal nonspherical noble metal nanoparticles. Advanced Materials 2010, 22, 1805-1825.

(29) Polte, J. Fundamental growth principles of colloidal metal nanoparticles - a new perspective. CrystEngComm 2015, 17, 6809-6830.

(30) Elsabahy, M.; Wooley, K. Design of polymeric nanoparticles for biomedical delivery applications. Chem. Soc. Rev. 2012, 41, 2545-2561.

(31) Zhang, G.; Niu, A.; Peng, S.; Jiang, M.; Tu, Y.; Li, M.; Wu, C. Formation of novel polymeric nanoparticles. Accounts of Chemical Research 2001, 34, 249-256.

(32) Peng, Z.; Liu, H. Bottom-up Nanofabrication Using DNA Nanostructures. Chemistry of Materials 2016, 28, 1012-1021. 
(33) Chandrasekaran, A. R.; Levchenko, O. DNA Nanocages. Chemistry of Materials 2016, 28, 5569-5581.

(34) Latreille, P. L.; Adibnia, V.; Nour, A.; Rabanel, J. M.; Lalloz, A.; Arlt, J.; Poon, W. C.; Hildgen, P.; Martinez, V. A.; Banquy, X. Spontaneous shrinking of soft nanoparticles boosts their diffusion in confined media. Nature Communications 2019, 10.

(35) Anselmo, A. C.; Zhang, M.; Kumar, S.; Vogus, D. R.; Menegatti, S.; Helgeson, M. E.; Mitragotri, S. Elasticity of nanoparticles influences their blood circulation, phagocytosis, endocytosis, and targeting. ACS Nano 2015, 9, 3169-3177.

(36) Miller, B.; Imel, A. E.; Holley, W.; Baskaran, D.; Mays, J. W.; Dadmun, M. D. The Role of Nanoparticle Rigidity on the Diffusion of Linear Polystyrene in a Polymer Nanocomposite. Macromolecules 2015, 48, 8369-8375.

(37) Imel, A. E.; Rostom, S.; Holley, W.; Baskaran, D.; Mays, J. W.; Dadmun, M. D. The tracer diffusion coefficient of soft nanoparticles in a linear polymer matrix. $R S C$ Advances 2017, 7, 15574-15581.

(38) Rostom, S.; Dadmun, M. D. The impact of nanoparticle softness on its tracer diffusion coefficient in all polymer nanocomposites. Journal of Applied Physics 2020, 127, 1-9.

(39) Hershberger, K. K.; Gauger, A. J.; Bronstein, L. M. Utilizing Stimuli Responsive Linkages to Engineer and Enhance Polymer Nanoparticle-Based Drug Delivery Platforms. ACS Applied Bio Materials 2021, 4, 4720-4736.

(40) Wechsler, M. E.; Vela Ramirez, J. E.; Peppas, N. A. 110th Anniversary: Nanoparticle Mediated Drug Delivery for the Treatment of Alzheimer's Disease: Crossing the BloodBrain Barrier. Industrial and Engineering Chemistry Research 2019, 58, 15079-15087.

(41) Cevaal, P. M.; Ali, A.; Czuba-Wojnilowicz, E.; Symons, J.; Lewin, S. R.; Cortez- 
Jugo, C.; Caruso, F. In Vivo T Cell-Targeting Nanoparticle Drug Delivery Systems: Considerations for Rational Design. ACS Nano 2021, 15, 3736-3753.

(42) Srinivasarao, M.; Low, P. S. Ligand-Targeted Drug Delivery. Chemical Reviews 2017, $117,12133-12164$.

(43) Gulati, N. M.; Stewart, P. L.; Steinmetz, N. F. Bioinspired Shielding Strategies for Nanoparticle Drug Delivery Applications. Molecular Pharmaceutics 2018, 15, 29002909.

(44) Argyo, C.; Weiss, V.; Bräuchle, C.; Bein, T. Multifunctional mesoporous silica nanoparticles as a universal platform for drug delivery. Chemistry of Materials 2014, 26, 435451.

(45) Kulshreshtha, A.; Jayaraman, A. Dispersion and Aggregation of Polymer Grafted Particles in Polymer Nanocomposites Driven by the Hardness and Size of the Grafted Layer Tuned by Attractive Graft-Matrix Interactions. Macromolecules 2020, 53, 1302-1313.

(46) Shrestha, U. M.; Han, L.; Saito, T.; Schweizer, K. S.; Dadmun, M. D. Mechanism of Soft Nanoparticle Diffusion in Entangled Polymer Melts. Macromolecules 2020, 53, $7580-7589$.

(47) Griffin, P. J.; Bocharova, V.; Middleton, L. R.; Composto, R. J.; Clarke, N.; Schweizer, K. S.; Winey, K. I. Influence of the Bound Polymer Layer on Nanoparticle Diffusion in Polymer Melts. ACS Macro Letters 2016, 5, 1141-1145.

(48) Poling-Skutvik, R.; Slim, A. H.; Narayanan, S.; Conrad, J. C.; Krishnamoorti, R. Soft interactions modify the diffusive dynamics of polymer-grafted nanoparticles in solutions of free polymer. ACS Macro Letters 2019, 8, 917-922.

(49) Kohli, I.; Mukhopadhyay, A. Di ff usion of Nanoparticles in Semidilute Polymer Solutions: E ff ect of Di ff erent Length Scales. Macromolecules 2012, 45, 6143-6149. 
(50) Senanayake, K. K.; Fakhrabadi, E. A.; Liberatore, M. W.; Mukhopadhyay, A. Diffusion of Nanoparticles in Entangled Poly(vinyl alcohol) Solutions and Gels. Macromolecules 2019, 52, 787-795.

(51) Yamamoto, U.; Schweizer, K. S. Microscopic theory of the long-time diffusivity and intermediate-time anomalous transport of a nanoparticle in polymer melts. Macromolecules 2015, 48, 152-163.

(52) Bailey, E. J.; Winey, K. I. Dynamics of polymer segments, polymer chains, and nanoparticles in polymer nanocomposite melts: A review. Progress in Polymer Science 2020, 105, 101242.

(53) Lorenzo, F. D.; Seiffert, S. Nanostructural heterogeneity in polymer networks and gels. Polymer Chemistry 2015, 6, 5515-5528.

(54) Danielsen, S. P. et al. Molecular Characterization of Polymer Networks. Chemical Reviews 2021, 121, 5042-5092.

(55) Tsuji, Y.; Li, X.; Shibayama, M. Evaluation of Mesh Size in Model Polymer Networks Consisting of Tetra-Arm and Linear Poly(ethylene glycol)s. Gels 2018, 4, 50.

(56) Gole, A.; Murphy, C. J. Seed-mediated synthesis of gold nanorods: Role of the size and nature of the seed. Chemistry of Materials 2004, 16, 3633-3640.

(57) Marbella, L. E.; Chevrier, D. M.; Tancini, P. D.; Shobayo, O.; Smith, A. M.; Johnston, K. A.; Andolina, C. M.; Zhang, P.; Mpourmpakis, G.; Millstone, J. E. Description and Role of Bimetallic Prenucleation Species in the Formation of Small Nanoparticle Alloys. Journal of the American Chemical Society 2015, 137, 15852-15858.

(58) Hettiaratchi, M. H.; Schudel, A.; Rouse, T.; García, A. J.; Thomas, S. N.; Guldberg, R. E.; McDevitt, T. C. A rapid method for determining protein diffusion through hydrogels for regenerative medicine applications. APL Bioengineering 2018, 2. 
(59) Oesterhelt, F.; Rief, M.; Gaub, H. E. Single molecule force spectroscopy by AFM indicates helical structure of poly(ethylene-glycol) in water. New Journal of Physics $1999,1,6-6$.

(60) Kotsmar, C.; Sells, T.; Taylor, N.; Liu, D. E.; Prausnitz, J. M.; Radke, C. J. Aqueous solute partitioning and mesh size in HEMA/MAA hydrogels. Macromolecules 2012, 45, 9177-9187. 


\section{TOC Graphic}

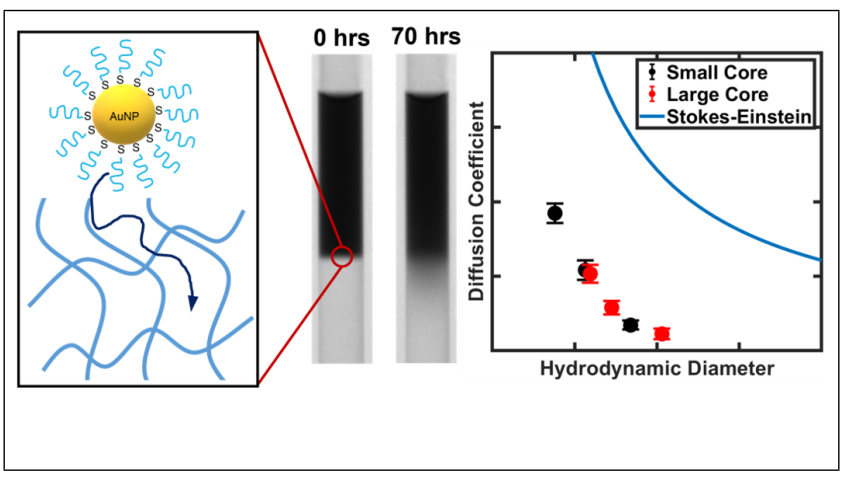

